\title{
EUSO-SPB1 Mission and Science
}

\section{Wiencke ${ }^{* 1} \dagger$ A. Olinto ${ }^{2}$ for the JEM-EUSO Collaboration}

${ }^{1}$ Colorado School of Mines

${ }^{2}$ University of Chicago

The Extreme Universe Space Observatory on a Super Pressure Balloon (EUSO-SPB1) was launched April 24th 23:51 UTC 2017 from Wanaka, New Zealand as a mission of opportunity on a NASA super pressure balloon test flight planned to circle the southern hemisphere. The primary scientific goal was to make the first observations of high energy cosmic ray extensive air showers by looking down on the atmosphere with a UV fluorescence detector from the near space altitude of $33 \mathrm{~km}$. After 12 days 4 hours aloft, the flight was terminated prematurely in the Pacific Ocean about $300 \mathrm{~km}$ SE of Easter Island. This overview presentation of the EUSO-SPB1 program will review the science of the mission, the instrument, the flight, and first looks at data. Preparations for a EUSO-SPB2 mission are underway.

35th International Cosmic Ray Conference - ICRC2017-

10-20 July, 2017

Bexco, Busan, Korea

\footnotetext{
* Speaker.

$\dagger$ lwiencke@mines.edu
} 


\section{Introduction}

The sources and acceleration mechanisms that produce the highest energy particles known to exist remain unknown. With energies that reach $100 \mathrm{EeV}$ and beyond, ultrahigh energy cosmic rays (UHECR) hold a tantalizing position in the multi-messenger view of the cosmos. They extend the energy range spanned by the various messengers upward by orders of magnitude. They are a different type of messenger that offers a new window on the Universe.

UHECRs that reach the earth's atmosphere convert their kinetic energy into extensive air showers (EAS). The low flux of UHECRs requires indirect measurement techniques that use the atmosphere as a giant calorimeter. The atmosphere is the largest volume calorimeter on Earth. The atmospheric fluorescence technique for measuring EASs in 3D has been developed and used on ground successfully by Fly's Eye [1], HiRes [2], the Pierre Auger Observatory [3] and Telescope Array [4].

EUSO-SPB1 is an important step in the challenging journey to a major UV fluorescence detector in space $[5-10]$ that would view a much larger $(\approx 100 \times)$ atmospheric footprint to measure UHECR EASs, map the entire sky, and discover the sources of UHECRs. Such a detector in space could also have sensitivity to high-energy photons, monopoles and super heavy forms of dark matter, and cosmogenic tau neutrinos.

The Extreme Universe Space Observatory on a Super Pressure Balloon (EUSO-SPB1) represents the collaboration's first mission to observe UHECR EASs by looking down on the atmosphere from sub-orbital space. This prototype instrument was flown as a mission of opportunity on a NASA super pressure balloon test flight that was launched on April 24th 23:51 UTC 2017 from Wanaka, New Zealand (Fig. 1). The mission science goals include:

- Make the first observations of Ultra High Energy Cosmic Ray Air Showers by looking down from suborbital space with an air fluorescence detector.

- Measure background UV light at night over ocean and clouds

- Search for fast UV pulse-like signatures from other objects

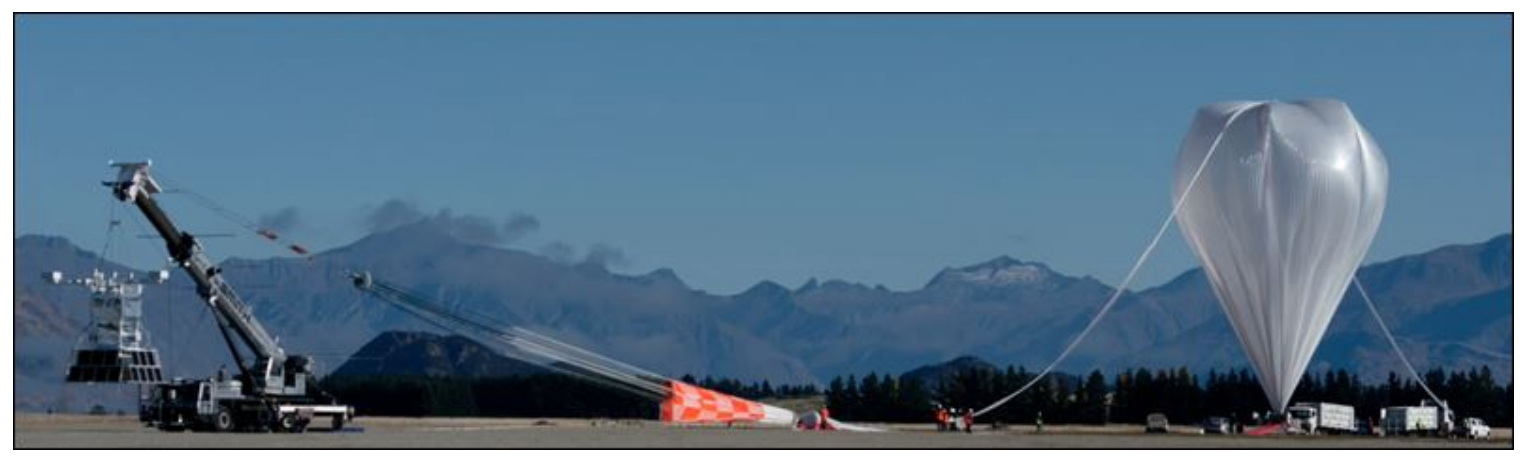

Figure 1: EUSO-SPB1 shortly before it was launched on April 24th 23:51 UTC 2017 from Wanaka NZ. The instrument is on the far left suspended from the launch crane. 


\section{Long Duration Scientific Balloon Flights from Wanaka NZ}

Located at $-45^{\circ}$ latitude, Wanaka NZ lies below a fast stratospheric air circulation that develops twice a year at about $33 \mathrm{~km}$ ( 7 mbar) above the southern ocean. This circulation flows easterly in the southern fall and westerly in the southern spring. A dynamic representation can be obtained from [11]. Super pressure balloons (SPBs) are designed to float at a constant displacement volume and consequently at a constant altitude. Thus a payload launched from Wanaka under a specially prepared SPB could ride this stratospheric circulation for months, complete an orbit every few weeks and terminate on land. In 2015 NASA launched an engineering SPB mission from Wanaka. It flew for 32 days and landed in Australia. The following year the first scientific payload flew for 46 days, circled the southern ocean, and landed in Peru. The 2017 flight was the 3rd SPB test flight from Wanaka. The duration could reach 100 days. Maps of these 3 flights are shown in Fig. 2.

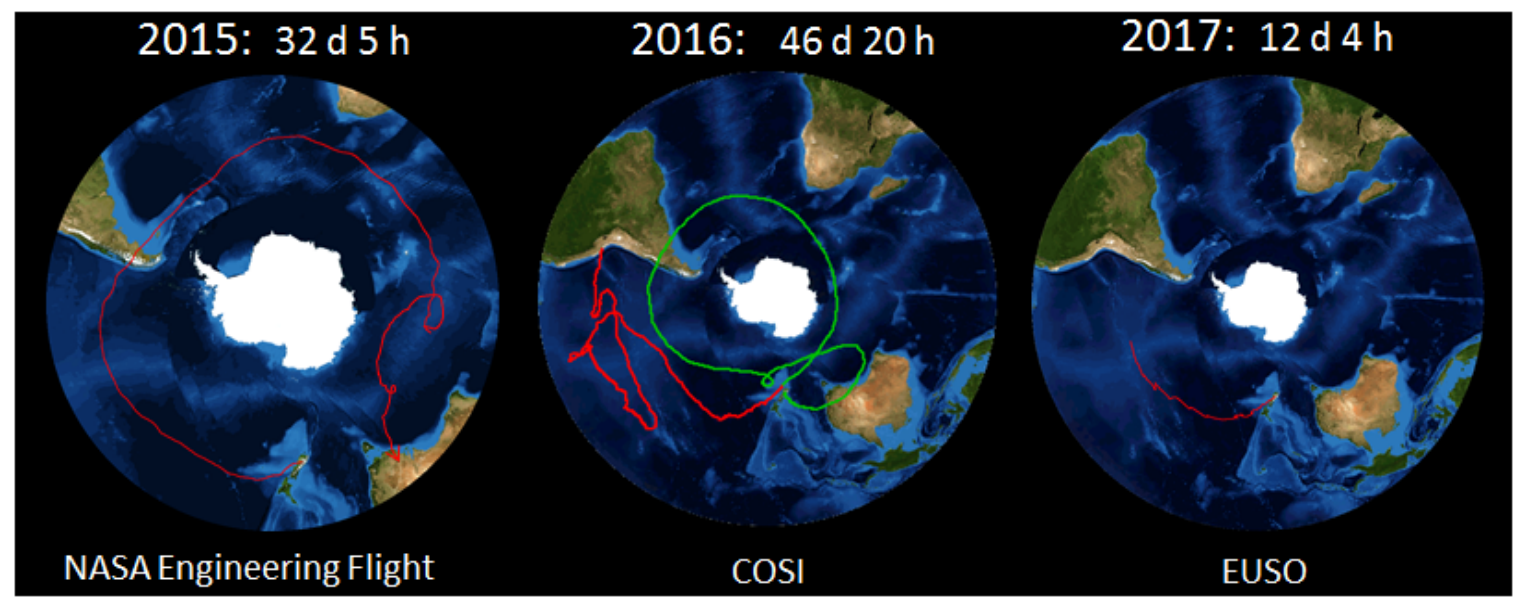

Figure 2: The trajectories of the NASA super pressure balloon test flights launched from Wanaka NZ.

Unfortunately the 2017 balloon developed an anomaly that lead to an early termination of the mission (Fig. 3). 12 days after launch the entire flight train had to be "valved down" into the ocean about $300 \mathrm{~km}$ SE of Easter Island.

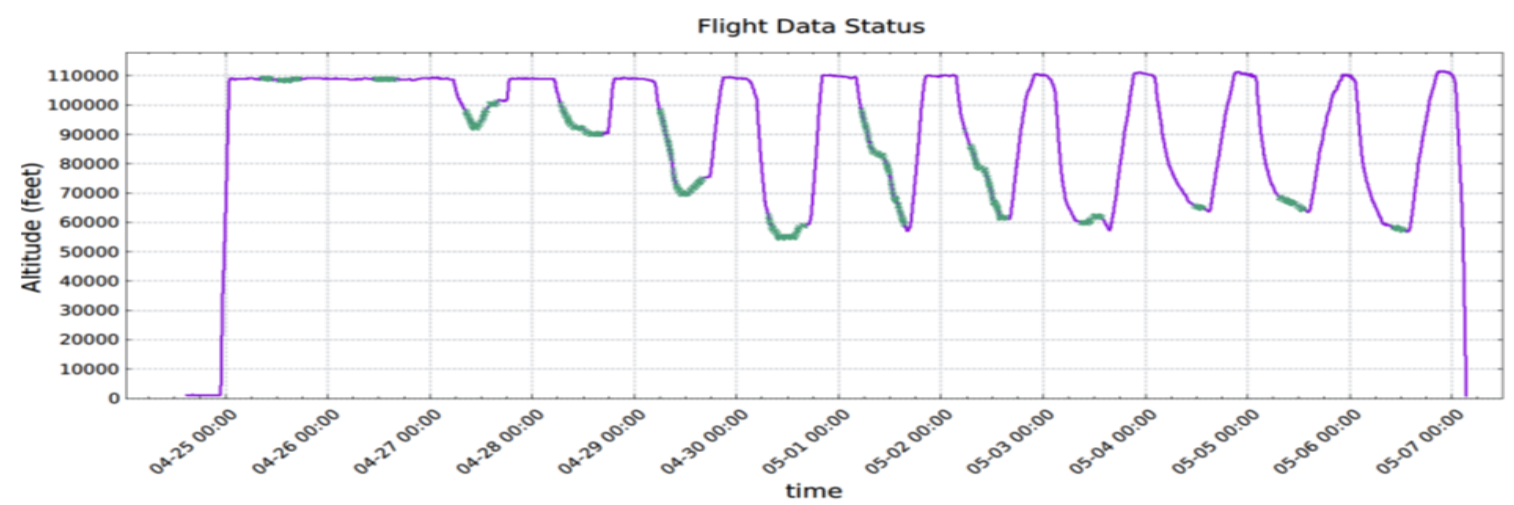

Figure 3: Altitude profile of the 2017 mission. The thicker lines denote the dark moonless periods when data was collected. 
Despite the setbacks, the EUSO-SPB1 instrument (Fig. 4) operated successfully while aloft and returned about $60 \mathrm{~GB}$ of data. Here we describe the instrument, preflight testing in the lab and desert, the mission, and provide some first looks at data. Planning and preparations for the EUSO-SPB2 mission (section 7) are underway.

\section{EUSO-SPB1 Instrument}

\subsection{4 instrument and mission}

The 2017 EUSO-SPB1 mission followed a 2014 EUSO-balloon overnight mission [12, 13] that was sponsored by the French Space Agency (CNES) and launched on a zero pressure balloon from Timmins Canada. During an 8 hour flight the UV camera was triggered externally by an onboard $20 \mathrm{~Hz}$ clock. The instrument recorded UV background light levels [14] and sampled UV flashes [15] and UV laser tracks [16] from a helicopter [17]. The helicopter, with flight crew, two students and light sources on board, circled below the balloon for 2 hours with the light sources firing. After the helicopter returned to base, the balloon drifted over a mining operation and the UV camera recorded, through chance coincidence, a flash with a timing shape consistent with that of a Xe flashlamp. The detector came down in a small lake and the instrument was recovered intact.

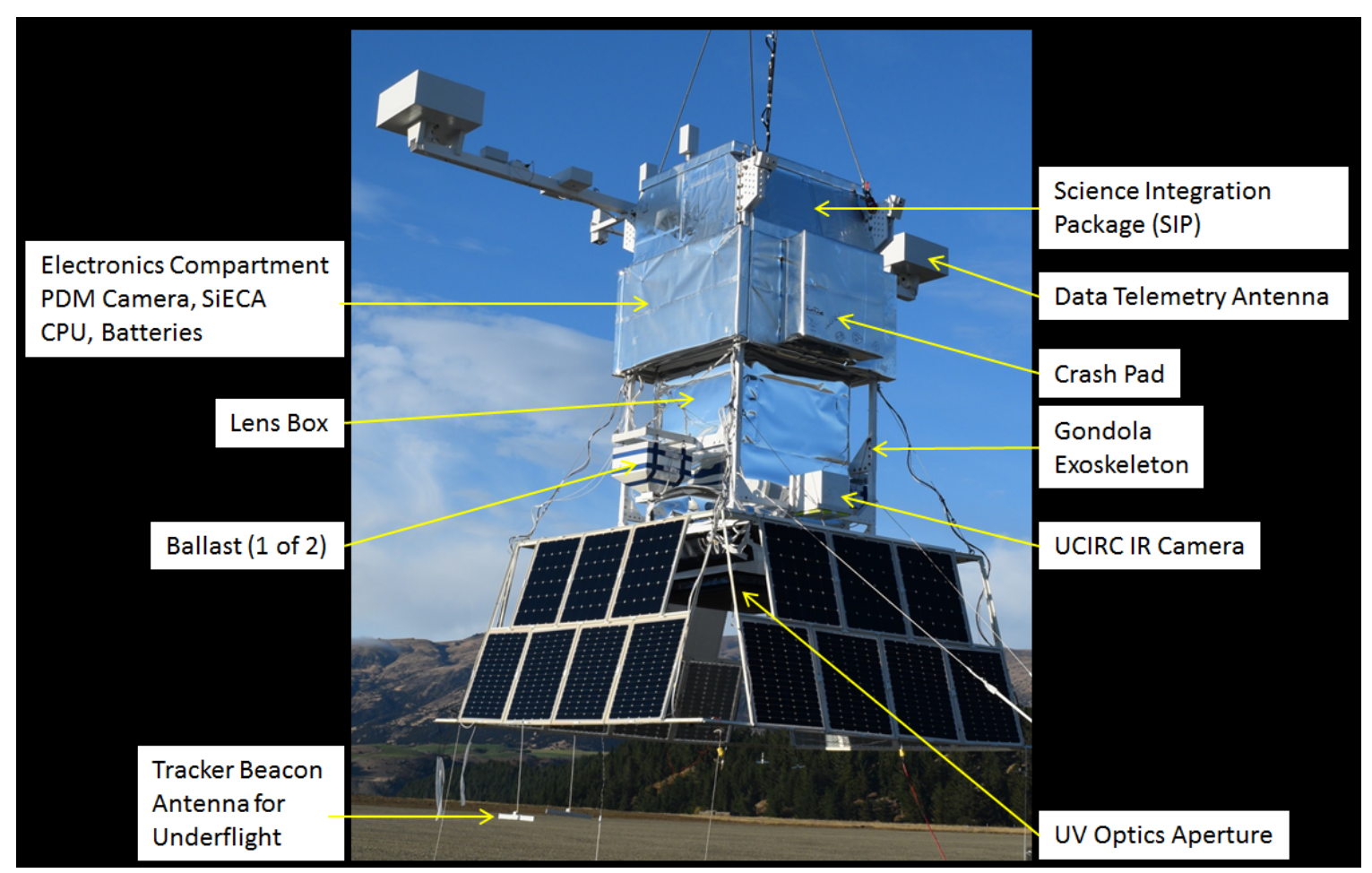

Figure 4: The EUSO-SPB1 instrument in flight ready configuration.

\subsection{7 instrument}

In preparation for the 2017 long duration flight, members of the JEM-EUSO collaboration upgraded the 2014 instrument extensively. Specifications of the 2017 instrument are listed in table 1. 
Upgrades included a new set of Fresnel lenses, a new UV camera (Fig. 5) with higher quantum efficiency multi-annode photomultiplier tubes (MAPMTs), an integrated high voltage (HV) system, a new flight CPU, a trigger to identify EAS candidates, interfaces to the NASA science integration package (SIP) and telemetry, new control software, a solar power system, and a gondola exoskeleton frame to which all equipment was mounted including an antenna boom.

\begin{tabular}{|c|c|c|}
\hline & Specification & Notes \\
\hline Energy Threshold & $\approx 3 \mathrm{EeV}$ & 50\% Trigger Threshold \\
\hline Trigger Aperture & $\begin{array}{l}\approx 250 \mathrm{~km}^{2} \mathrm{sr} @ 3 \mathrm{EeV} \\
\approx 500 \mathrm{~km}^{2} \mathrm{sr} @ 10 \mathrm{EeV}\end{array}$ & \\
\hline Telescope Optics & $21 \mathrm{~m}^{2}$ Fresnel Lenses & PMMA \\
\hline Field of View & $11.1^{\circ} \times 11.1^{\circ}$ & from stars, lasers \\
\hline Number of Pixels & $2304(48 \times 48)$ & $3664 \mathrm{ch}$. MAPMTs \\
\hline MAPMT & R11265-113-M64-MOD2 & Hamamatsu \\
\hline UV Filter & BG-3, $2 \mathrm{~mm}$ thick & 1 per MAPMT \\
\hline Read Out & DC coupled & $100 \mathrm{MHz}$ double pulse resolution \\
\hline Time Bin Duration & $2.5 \mu \mathrm{s}$ integration & event packet $=128$ bins $(320 \mu \mathrm{s})$ \\
\hline Flight CPU & CMA24GSD1000HR-4096 & RTD Technologies \\
\hline Telemetry (Data) & $2 \times \approx 75 \mathrm{kbits} / \mathrm{s}$ & 2 Irridium OpenPort \\
\hline Telemetry (Coms) & $\approx 1.2 \mathrm{kbits} / \mathrm{s}$ ( 255 bit bursts) & 2 Irridium Pilots \\
\hline Power Consumption & $40 \mathrm{~W}$ (day) $70 \mathrm{~W}$ (night) & includes $20 \mathrm{~W}$ heater \\
\hline Batteries & $10(12 \mathrm{~V}, 42 \mathrm{Ah}$, lead acid $)$ & Odyssey PC1200 \\
\hline Solar Panels & $3 \times 100 \mathrm{~W}$ on all 4 sides & SunCat Solar \\
\hline Detector Weight & $2700 \mathrm{lbs}(1227 \mathrm{~kg})$ & $2250 \mathrm{lbs}$ w/o SIP, Antennas \\
\hline Balloon & $18 \times 10^{6} \mathrm{ft}^{3} \quad\left(0.5 \times 10^{6} \mathrm{~m}^{3}\right)$ & helium \\
\hline Nominal Float Height & $110000 \mathrm{ft}(33.5 \mathrm{~km})$ & \\
\hline Releasable Ballast & $1200 \mathrm{lbs}(545 \mathrm{~kg})$ & $\leq 50 \mathrm{lbs}$ at termination \\
\hline Flight Start & April 24 23:51 UTC 2017 & -44.7218 lat +169.2540 long \\
\hline Flight End & May $6 \quad 3: 40$ UTC 2017 & -29.3778 lat -106.5037 long \\
\hline Flight Duration & 12 days 4 hours & \\
\hline
\end{tabular}

Table 1: Specifications of EUSO-SPB1 and 2017 mission.

EUSO-SPB1 was designed to operate at stratospheric altitude during nights with little or no moon and detect UV tracks from EASs. The instrument is detailed in these proceedings [18] and the subsystem architecture is diagrammed in Fig. 6. Two $1 \mathrm{~m}^{2}$ PMMA plastic Fresnel lenses focus light from below onto a UV sensitive high speed "camera". The camera focal surface features a Photo Detection Module (PDM) that counts single photoelectrons. For assembly purposes 4 MAPMTS of 64 channels, each covered by a BG-3 UV transmitting optical filter, are arranged to form a square "elementary cell" (EC). A PDM includes a $3 \times 3$ arrangement of 9 ECs. (For reference, the focal surface of the JEM-EUSO detector as designed for the International Space Station included 137 PDMs.) Located in the PDM behind the ECs are 6 digitization circuit boards. These plug into a central control buffer board that hosts the VHDL trigger logic.

The PDM is operated with $+1100 \mathrm{~V}$ applied to the photocathodes. The nominal gain is $10^{6}$. 

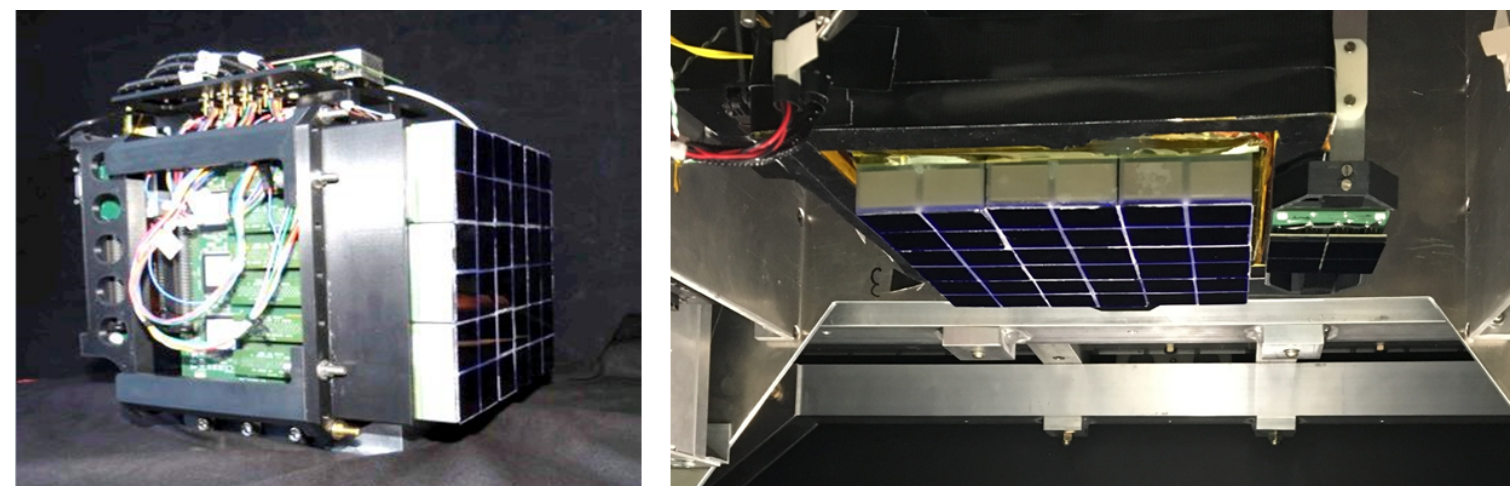

Figure 5: Left: The photo detection module (PDM) of the EUSO-SPB1 instrument: Right: As installed at the focal plane. The SiPM prototype cell is positioned to the right of the PDM.

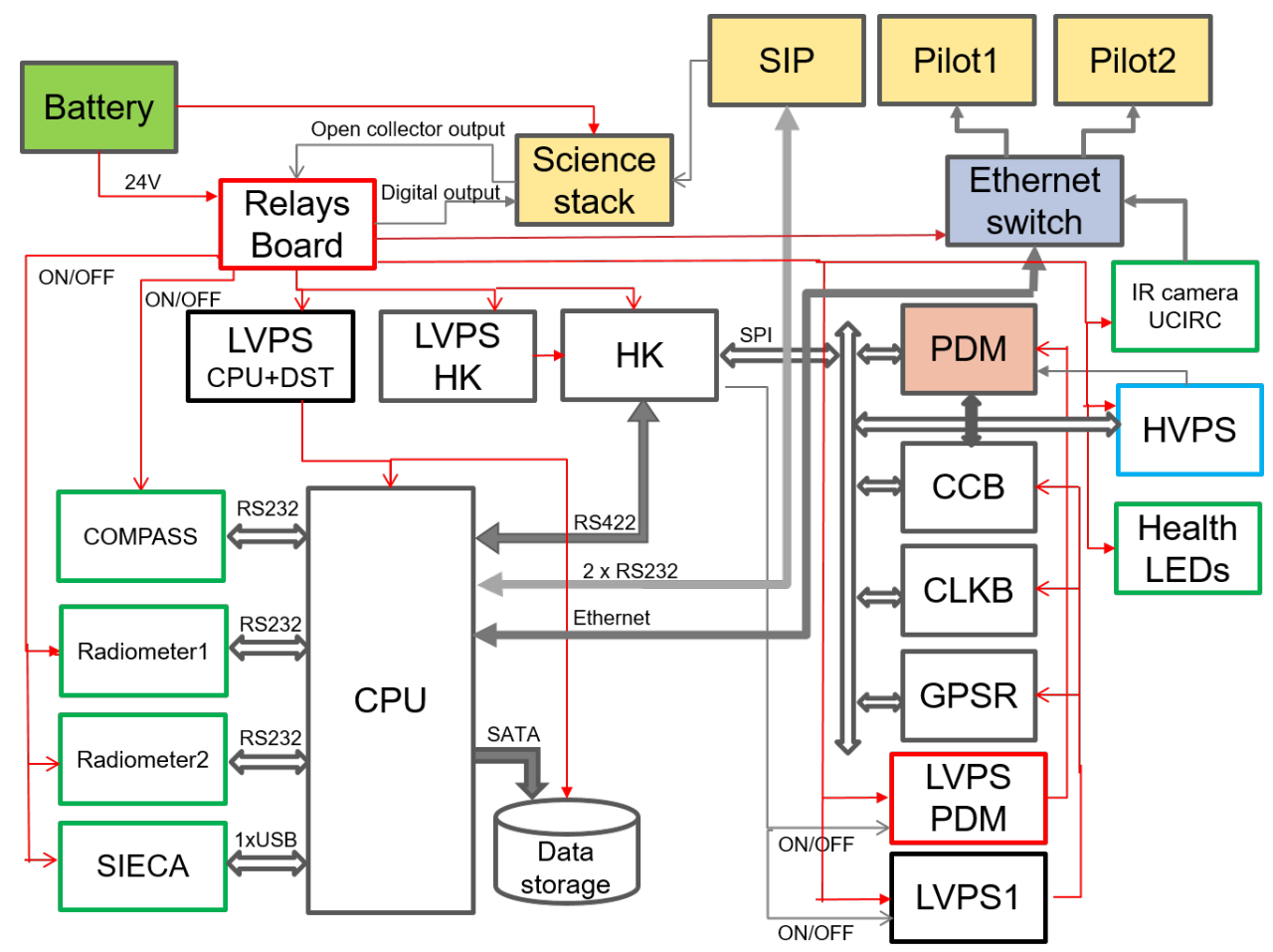

Figure 6: The EUSO-SPB1 data readout system and its connections to the various subsystems.

The HV for the MAPMTs is generated by a Cockcroft-Walton type circuit [19]. A corresponding circuit board and its HV connections with the MAPMTs are potted into each EC sub-assembly. The anodes of the MAPMTs are held at ground and coupled directly to the digitization electronics. The latter has been designed to count the small individual current pulses initiated by the emission of single photoelectrons from the photocathodes. The double pulse resolution is $100 \mathrm{MHz}$ (10 ns). The DC coupling permits, for example, photometric calibrations using pulsed and DC light sources and direct measurements of background light levels.

The on-board trigger system [20,21] operates at the MAPMT level and looks for locally persistent signals above background within $3 \times 3$ pixel cells. The local background threshold is adjusted 
dynamically to reduce significantly the number of fake triggers caused by slowly moving objects like stars, airplanes, and lightning. Field tests of the trigger system are described in section 4. Upon receipt of a trigger, the data processing (DP) system [22] copies 128 consecutive data frames (320 $\mu \mathrm{s}$ ) from a system buffer to an onboard $1 \mathrm{~TB}$ raid array. A single data frame contains a list of the number of photoelectrons recorded in each of the 2304 individual pixels over the same $2.5 \mu \mathrm{s}$ interval. The DP is also interfaced to the various ancillary systems described below. It includes a comprehensive modular flight control software package that can be readily configured for testing and flight.

As an R\&D test, the EUSO-SPB1 focal surface also include a 256 channel silicon photo sensor array [23] that was mounted next to the MAPMTs of the PDM (Figs. 5 and 7). This add-on system, called SiECA, was flown in a stand alone sampling mode.
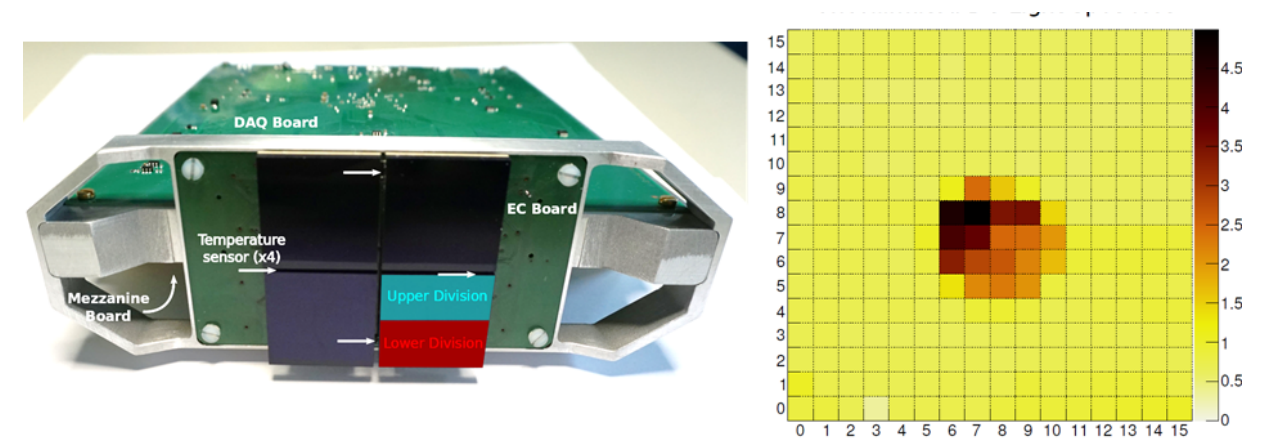

Figure 7: Left: This 256 channel silicon photomultipler array of 464 channel Hamamatsu S13361-3050AS08 sensors was flown as a test on the EUSO-SPB1 mission. Right: Lab data recorded from an uncollimated DC light spot source.

An IR camera system was developed and flown to record IR images of the scene below the balloon to identify clouds and estimate the heights of the cloud tops. Measuring clouds is important for estimating the exposure of EUSO-SPB1 because high clouds reduce the available aperture of the fluorescence telescope [24]. The University of Chicago Infra Red Camera (UCIRC) [25] featured two identical IR cameras that pointed down on the same region. The field of view was $24^{\circ} \times 30^{\circ}$. Each camera had an IR filter. One of the filters transmitted IR light between 11.5 and $12.9 \mu \mathrm{m}$ and the other transmitted IR light between 9.6 and $11.6 \mu \mathrm{m}$. These values were selected because they fall near the typical black body peak for clouds. The method to measure cloud color temperature from which the cloud top height can be derived is described in [26].

The mechanical upgrades to the payload complied with NASA requirements [27] for balloon gondolas. Additionally, the overall height, including antennas, had to be low enough to roll under the doorway of the aircraft hanger from which the mission was staged. The NASA SIP and antennas were mounted on top of the gondola frame and two ballast hoppers were mounted on opposite sides of the fame to allow the UV fluorescence telescope and IR camera system an unobstructed field of view looking down. The fluorescence telescope module could be rolled into the gondola frame structure and attached in about 30 minutes. The 4-sided solar array and a light baffle were connected to the payload outdoors following NASA safety procedures. 


\section{Field Test in the Utah Desert}

Prior to qualification testing at NASA's facility in Palestine TX, a team of collaborators transported the fully assembled fluorescence telescope system and a roving UV laser system [28,29] in two customized trailers from the assembly lab in Golden Colorado to the Telescope Array site in the Utah desert for field tests. Preparations for this $1600 \mathrm{~km}$ round trip were extensive [30]. Precautions included carrying the flight instrument on a vibration damped custom dolly inside an air ride trailer, driving a dummy load over the planned route first, logging both trips with accelerometers, and using a pilot vehicle. Data logged by the accelerometer on the flight instrument during the trip to the desert was mostly below $0.5 \mathrm{~g}$ with occasional bigger bumps (Fig. 8). (For reference, NASA requires that flight ready balloon payloads must be able to tolerate jolts of $0.5 \mathrm{~g}$ during transfer to the launch pad.) In the desert the trailer served as the field enclosure for EUSO-SPB1 and was positioned next to the EUSO-TA prototype [31] for coincident observations. The dolly was tilted up to point the detector's optical axis about $8^{\circ}$ above the horizon. Four hours after arriving in the desert, the EUSO-SPB1 detector turned on smoothly and recorded first tracks from lasers.

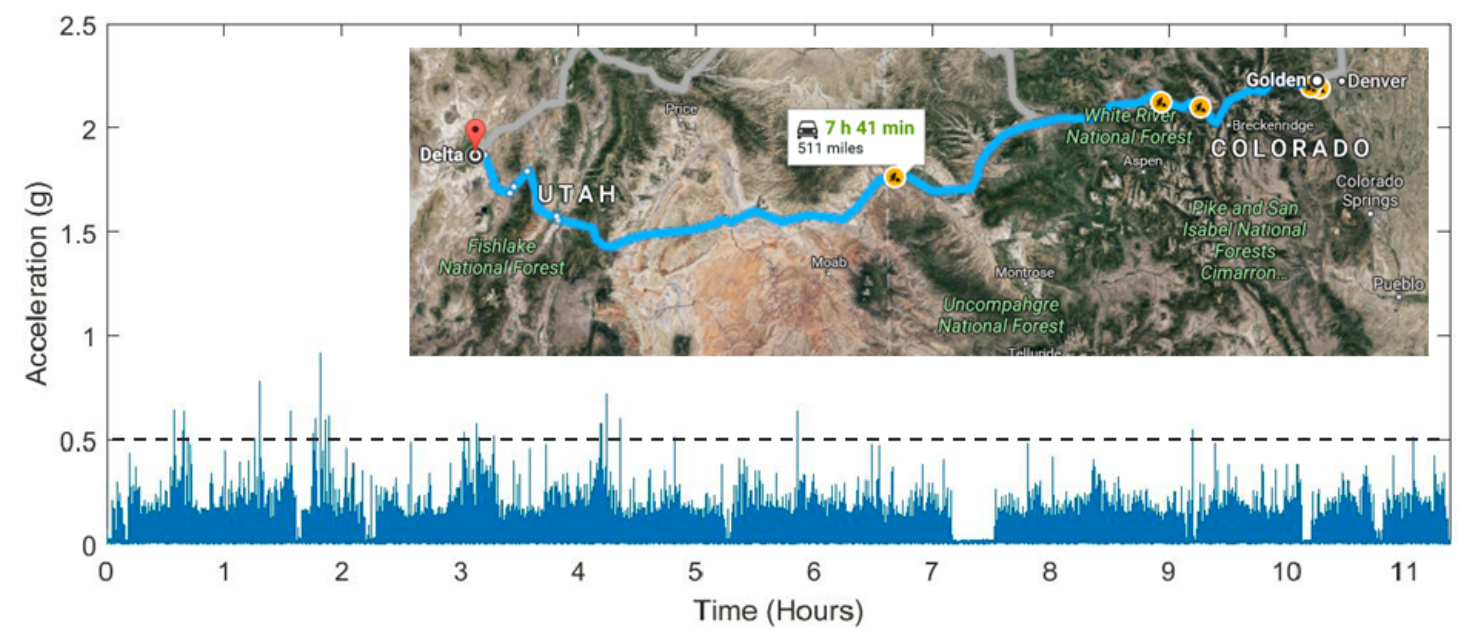

Figure 8: Data logged by an accelerometer that was attached to the assembled fluorescence telescope for the drive from the Golden $\mathrm{CO}$ assembly lab to the Utah desert for field tests. The dashed line at $0.5 \mathrm{~g}$ represents a NASA requirement (see text). Higher spikes were typically encountered on highway bridge joints.

Over the 6 day desert campaign the EUSO-SPB1 fluorescence telescope recorded about 100,000 laser shots and 1000s of flashes from a calibrated UV LED on a mast. A laser pulse crossing the FOV is shown in Fig. 9. Light flashes from commercial aircraft and slowly moving spots from stars and meteorites were also observed.

The fluorescence telescope field of view (FOV) was measured independently using stars and laser tracks (Fig. 10). The laser beam direction was swept across the vertical plane perpendicular to the vector between the laser and detector site. FOV measurements of $11.10 \pm 0.15^{\circ}$ were obtained from stars and $11.2 \pm 0.1^{\circ}$ were obtained from laser tracks.

From the measurements of the calibrated UV LEDs on a mast at near field distance, an absolute photometric calibration of $0.08 \pm 0.01$ photoelectrons per incident photon was obtained [32] for the fluorescence telescope. This preliminary result is consistent with the value of $0.09 \pm 0.01$ that was 


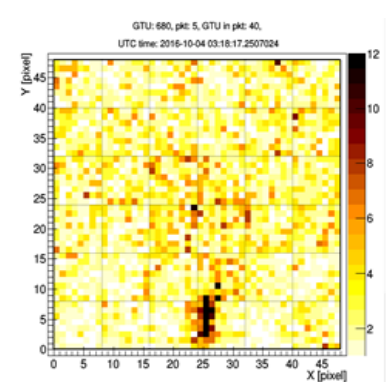

GTU 680

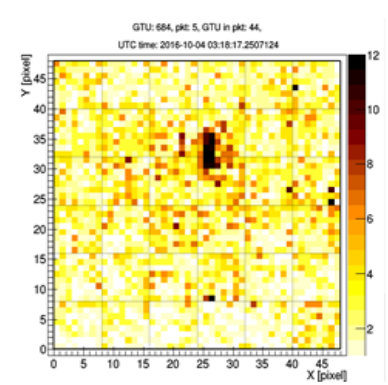

GTU 684

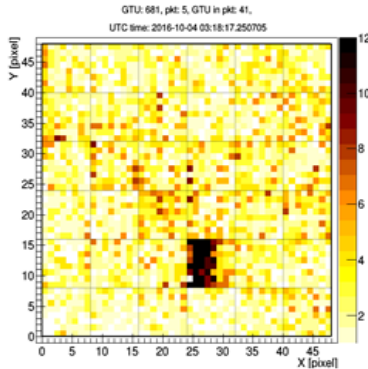

GTU 681

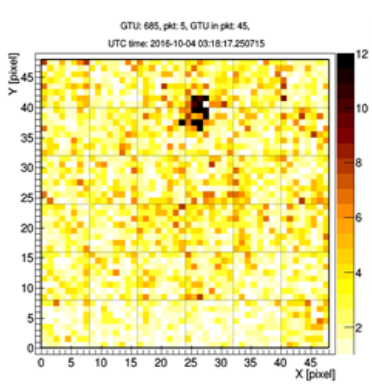

GTU 685

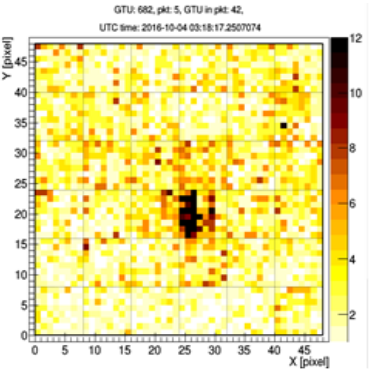

GTU 682

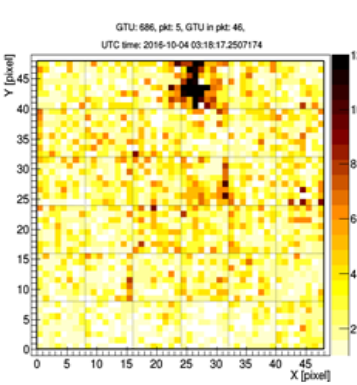

GTU 686

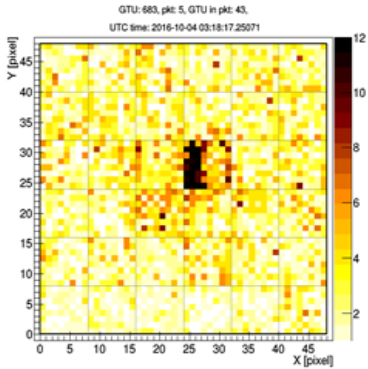

GTU 683

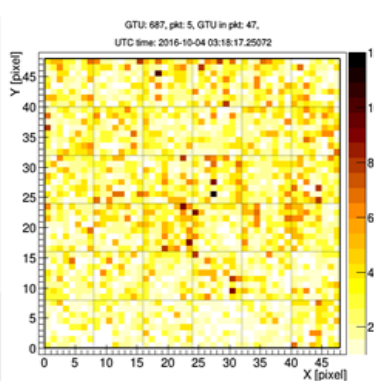

GTU 687

Figure 9: During desert testing a laser track propagates upward across the EUSO-SPB1 detector field of view. Each of the 8 frames shows a $2.5 \mu$ s exposure.

obtained by multiplying the laboratory measurement of the Fresnel 2-lens system efficiency with the laboratory measurements of photometric calibration of the PDM.

Measurements of laser tracks were used to estimate the efficiency of the on-board trigger using a persistence setting of 2 GTUs. Efficiency was measured by sweeping the laser in energy and counting how many tracks were recorded per 100 shots fired at each energy setting. For this study the laser was placed $24 \mathrm{~km}$ from the detector and the beam steering optics were aimed $45^{\circ}$ away from the detector. This distance and relative angle between fluorescence telescope and the laser were selected because they were representative of the distance and the typical relative angle at which the EUSO-SPB1 fluorescence telescope could be expected to observe EASs while looking down on the troposphere during the balloon flight.

Noise trigger rates were typically at the level of $1 \mathrm{~Hz}$ over the full PDM during the field tests. These trigger rates rose slightly when bright stars or meteorites crossed the field of view, or when there was lightning outside the field of view. A more extensive discussion is available in [20].

This efficiency study using the laser was performed for 2 configurations of the fluorescence telescope optics. The baseline configuration used 2 refractive Fresnel lenses. The other ( 3 lens) configuration included a diffractive middle lens for chromatic correction. The data points from the efficiency measurements, after noise triggers were removed, are plotted in Fig. 11. A laser energy of $0.8 \mathrm{~mJ}$ yielded a 50\% trigger efficiency for the 2 lens configuration. For the 3 lens configuration, despite its smaller spot size, the laser energy required for $50 \%$ trigger efficiency was about a factor of 2 higher. This factor of 2 was consistent with laboratory measurements [32] of the relative optical efficiency of the 2 and 3 lens configurations. Following these measurements it was decided to fly the 2 lens configuration.

The EAS energy corresponding to the UV laser energy was estimated in terms of optical 


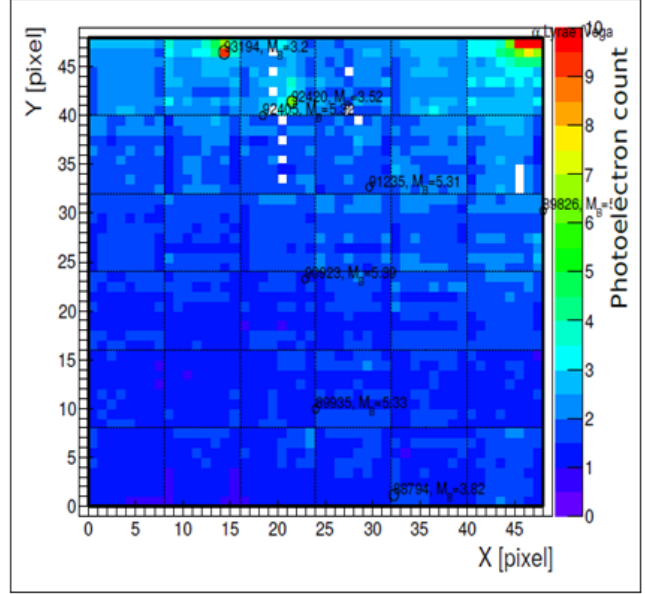

Stars

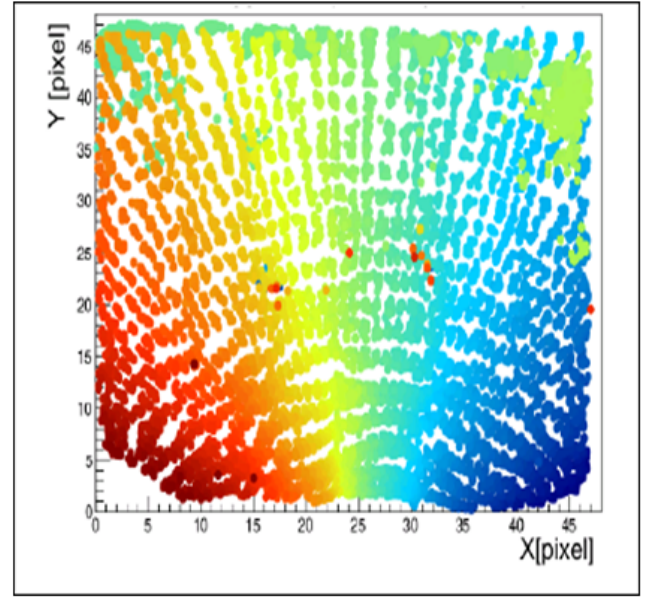

Laser

Figure 10: Left: Summed images from EUSO-SPB1 during desert ground tests shows images of stars above background. Right: A sweep of tracks as a laser beam is swept across the field of view.

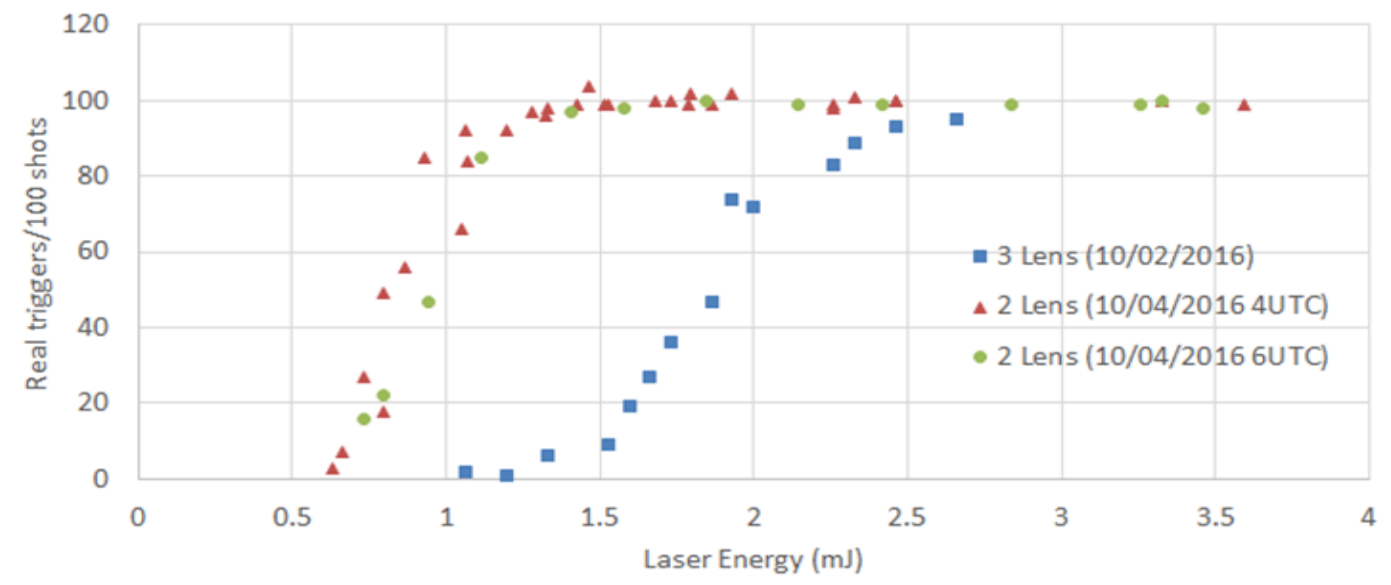

Figure 11: Trigger efficiencies for different lens configurations of the fluorescence telescope as measured by sweeping the energy of a distant UV laser. 100 shots were fired at each energy setting. See text for details.

equivalence. Assuming a nominal aerosol optical depth for the Utah fall desert, and using the laser and detector geometry of the field tests, the amount of light reaching the EUSO-SPB1 fluorescence detector was estimated for a $0.8 \mathrm{~mJ} 355 \mathrm{~nm}$ laser pulse. Using simulations, an EAS from a cosmic ray of about $3 \mathrm{EeV}$ was estimated to generate a similar amount of light at the EUSO-SPB1 detector for the case that the detector was pointing down on the atmosphere from the planned $33 \mathrm{~km}$ float altitude of the balloon. In other words, the inclined $0.8 \mathrm{~mJ}$ laser, as viewed from the side in the desert test configuration, was estimated to appear about as bright as an inclined 3 EeV EAS would appear when the EAS was viewed looking down from the float altitude of the balloon. 


\section{EUSO-SPB1 Campaign}

\subsection{Preparation and Launch}

The instrument departed Palestine TX Dec 2nd and reached the Wanaka NZ airport launch site in late January 2017 where it was unpacked, reassembled. Based on a thermal model study, removable thermal insulation panels were mounted around the vertical sides of electronics compartment. The instrument was suspended from a crane at night above an illuminated Tyvek screen for a final flat field calibration. After passing compatibility and hang tests, the instrument was declared flight ready on March 25th 2017.

The launch occurred on April 24th 23:30 UTC one day before the new moon. After reaching the New Zealand coast, the balloon traveled northward passing about $30 \mathrm{~km}$ east of Christchurch before heading out over the Pacific ocean. A 24/7 shift schedule for EUSO-SPB1 instrument monitoring and operations was setup with 6 centers in Europe (France, Germany, Italy), RIKEN Japan, and Colorado US. A Cesna 421C aircraft to fly LEDs and a laser under the balloon after one orbit was in preparation [33]. During the flight 40 hours of PDM data were recorded over 11 nights. To facilitate downloading, data files were restarted automatically every 2 minutes. A shorter file was recorded at the start of each hour and downloaded with highest priority to provide detector monitoring. The data download bandwidth was reduced when 1 of the 2 links failed. 30 hours of the data recorded on board were downloaded.

\section{First Look at Data}

To monitor the PDM and readout, a UV "Health" LED mounted in front of the PDM (Fig. 12 right panel) was fired twice every 16 seconds. An example pulse sequence from the Health LED and recorded by the PDM in flight is shown in Fig. 12 (left panel). The sample of the LED measurements downloaded over the flight demonstrates the stability of the detector response to the LED (Fig. 13). Most of the data points fall within $\pm 5 \%$ of the mean despite night time temperature swings of $30 \mathrm{C}^{\circ}$ outside the detector.
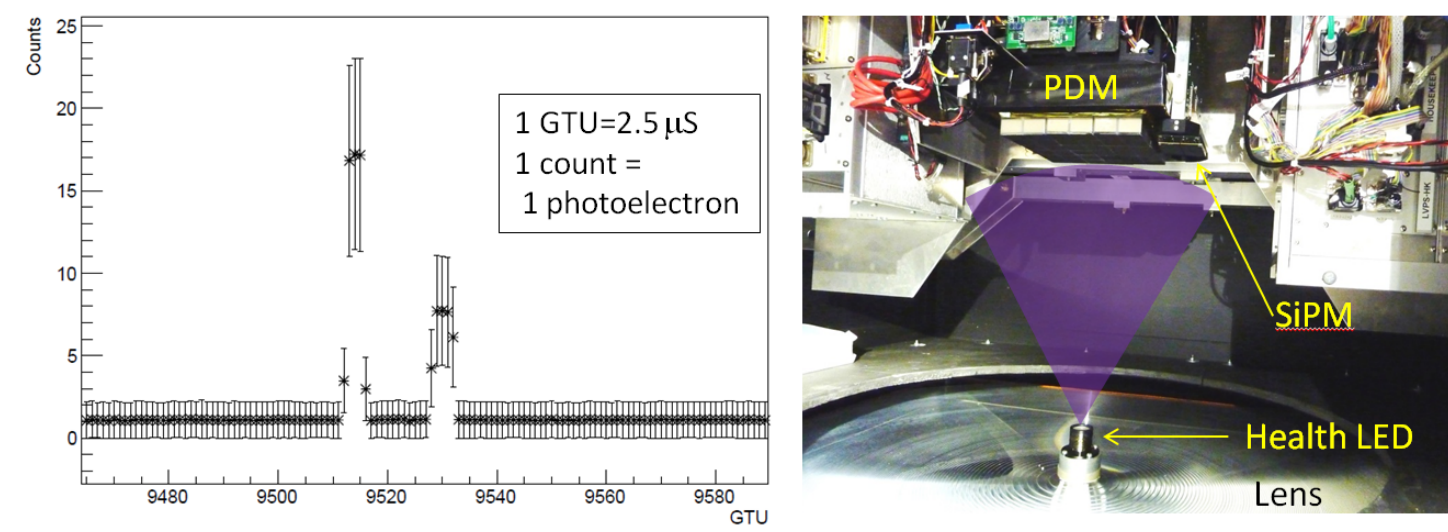

Figure 12: Left: A double pulse generated by the on board Health LED system and recorded by EUSOSPB1. The response shown here is the average over all pixels. Right: The location of the Health LED in the center of the back lens inside the electronics compartment of the instrument. 


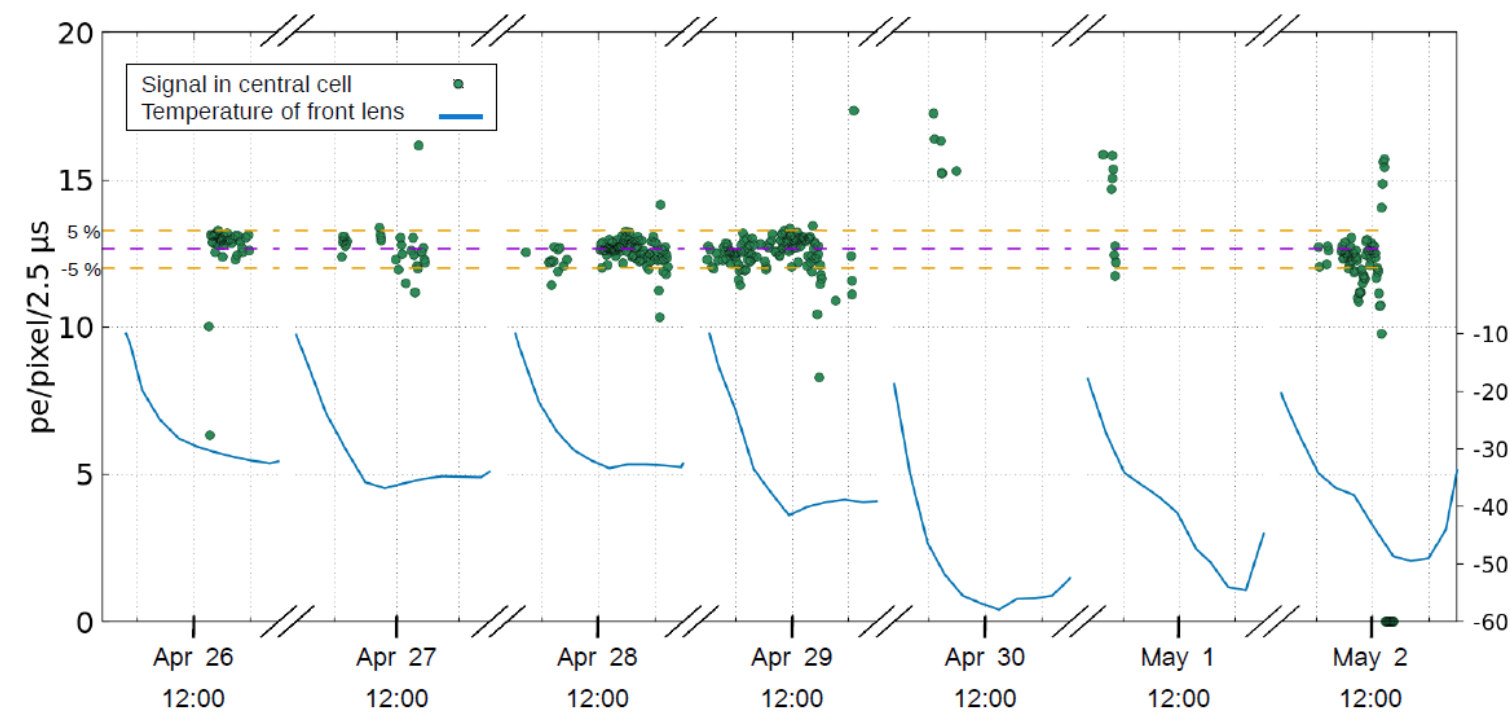

Figure 13: The response of the central cell of the EUSO-SPB1 PDM to flashes from the Health LED (dots). The dashed lines indicate $\pm 5 \%$ about the mean. The lower curves show the temperature measured at the outside of the front Fresnel lens at the optics entrance aperture. Dates and times are in UTC. Some gaps in the data occurred because the LED was turned off to avoid RF pickup from an onboard radio beacon that was enabled when the balloon dropped below 70,000 ft.

Data from the IR camera system during the ascent are shown in Fig. 14. Information about the presence of clouds in the PDM FOV can also be obtained from the PDM data. Variations in the average background rate of the fluorescence telescope PDM can show clouds passing under the balloon as seen in Fig. 15.
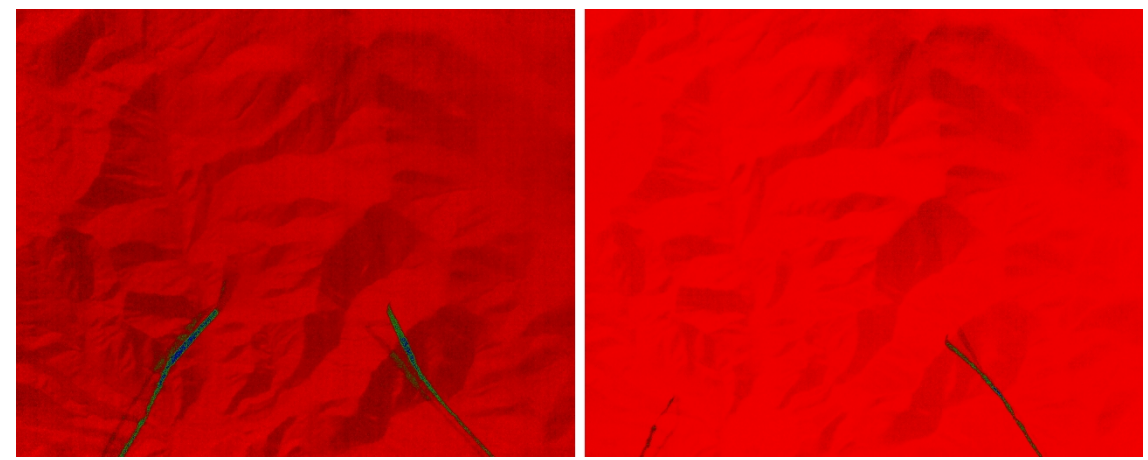

Figure 14: Temperature calibrated IR images of the Southern Alps taken by UCIRC while EUSO-SPB1 ascended over the southern island of New Zealand. Left: 11.5-12.9 $\mu \mathrm{m}$ filter Right: 9.6-11.6 $\mu \mathrm{m}$ filter.

The average background rates and trigger rates recorded during the mission are displayed in Fig. 16. The trigger rates during the first three nights occurred while the PDM was operating with the 1 GTU persistence trigger setting. When the persistence setting was changed to 2 GTU the rate dropped to a few $\mathrm{Hz}$ or lower. 

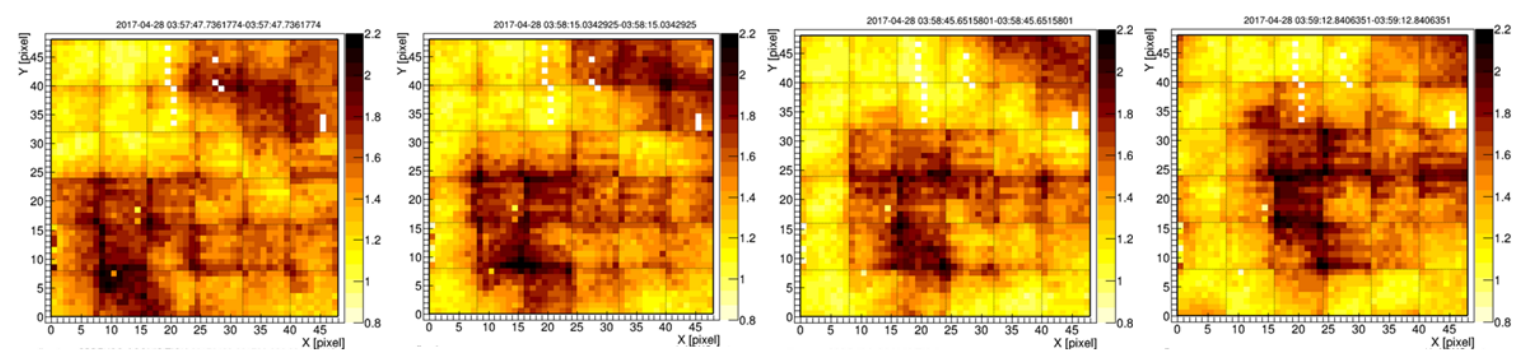

Figure 15: Example images of clouds drifting across the field of view of the UV PDM camera recorded April 28 2017. Each image represents the average of about $10002.5 \mu$ s images. The time between panels is about 30 seconds. The dark regions are clouds and the observed average counts from these regions are about 2 photoelectons/pixel/2.5 $\mu \mathrm{s}$. The average count rates in the light colored regions are about half this amount.
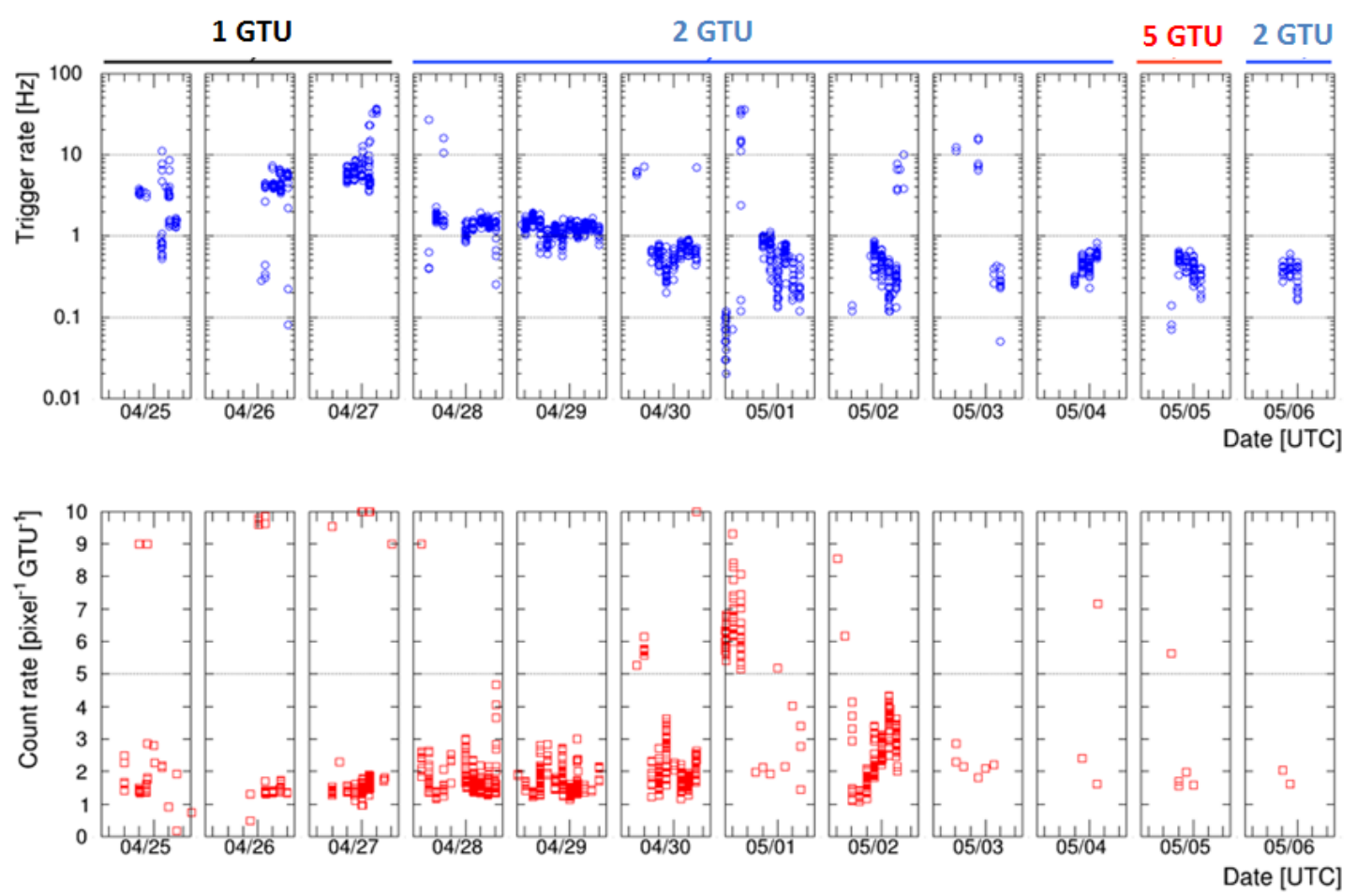

Figure 16: Top Panel: The trigger rate measured during the mission. The trigger persistence setting is listed above. Lower Panel: Preliminary measurement of the night time background light levels recorded during the mission. The units are photoelectrons/pixel/2.5 $\mu$ s. From ref [20].

One source of background triggers are very low energy cosmic rays that strike the PDM directly. An example of one of these can be seen in the sequence of images in Fig. 17. These triggers are distinct from an EAS signature because the direct track crosses the entire PDM within a single $2.5 \mu$ s time frame. An EAS viewed from the balloon can not do this.

The search for EAS candidates is in progress. The number of cosmic ray air showers expected has been derived from two independent simulations [34] and [30,35]. A simulated energy distribution, based on a longer duration SPB flight than was realized, is shown in Fig. 18. When scaled to the duration of the mission flown and assuming a $50 \%$ cloud obscuration the number of EAS 

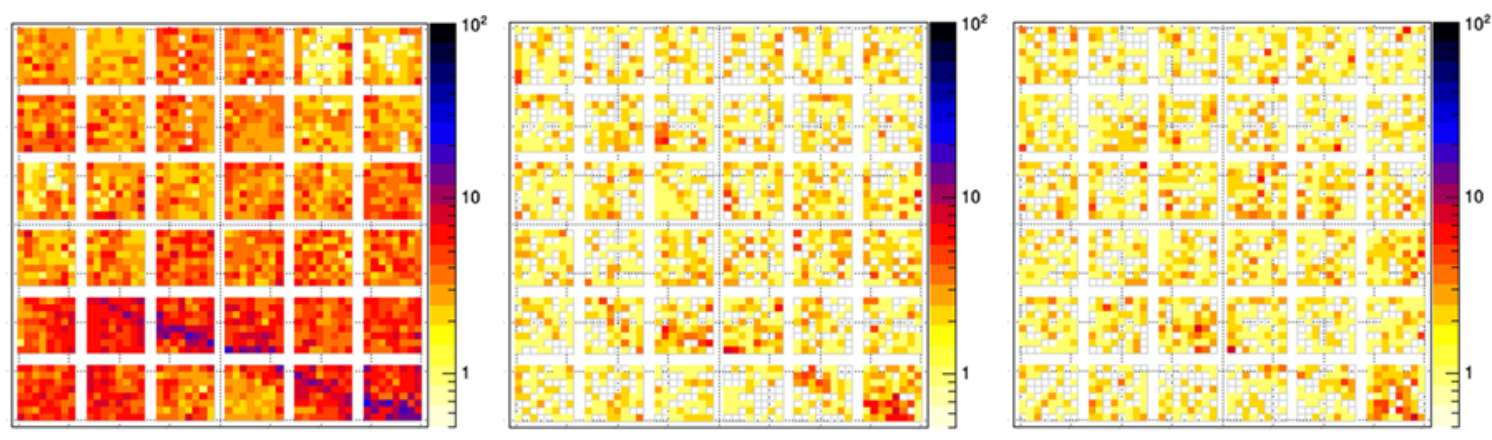

Figure 17: A direct cosmic ray traveling through the PDM camera. The 3 panels correspond to 3 consecutive $2.5 \mu$ s time bins.

events expected is about 1 . Updates to the simulations are in progress to include the lower altitude of the instrument during nighttime data taking.

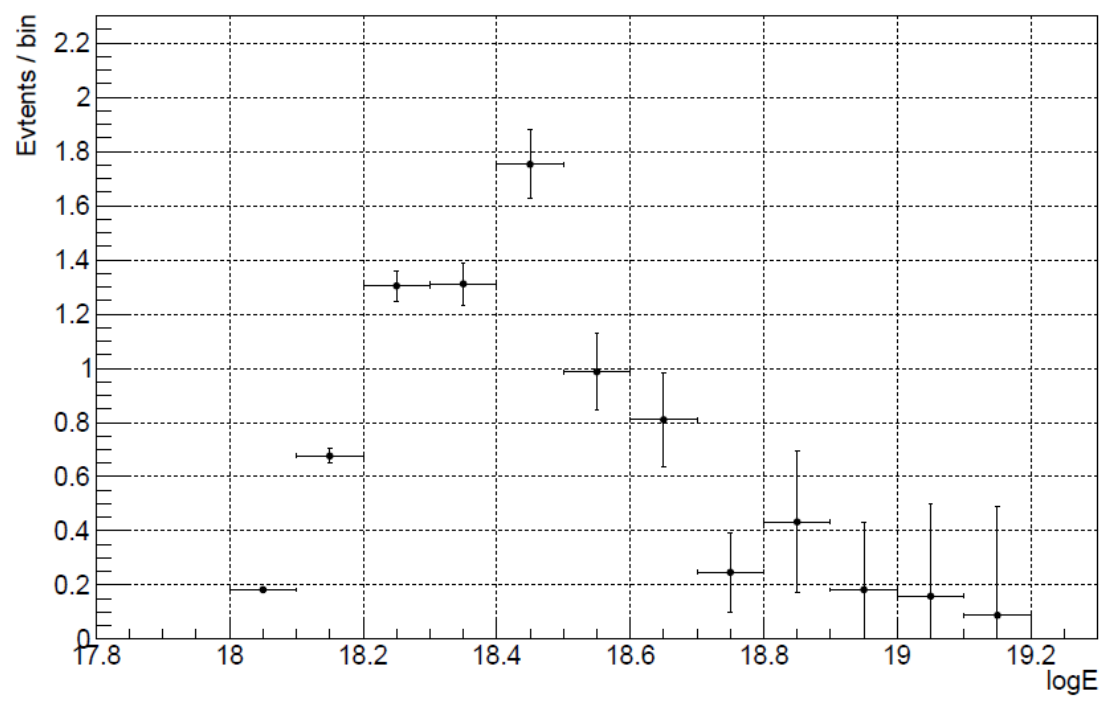

Figure 18: Expected number of triggered EAS events assuming a 118 hour clear dark period and constant $33 \mathrm{~km}$ altitude. (From reference [34]) When scaled for the parameters of the $2017 \mathrm{flight}$, the expected number of EASs is about 1.

\section{EUSO-SPB2}

Preparations are underway for a EUSO-SPB2 mission [36] that would expand the science goals of EUSO-SPB1 in support of a future space based UHECR observatory. The primary target of support is the Probe of Extreme Energy Multi Messenger Astronomy (POEMMA) mission [10] that is currently under a NASA sponsored study. Planned improvements in EUSO-SPB2 include higher efficiency optics to decrease the EAS energy threshold, a shorter integration time on the PDM camera to reduce background light exposure, a larger test SiPM array with faster timing, 
and a tilting mechanism to steer the telescope from nadir to $\approx 10^{\circ}$ above the horizon. This inflight tilting would enable, for example, a nadir mode as used in EUSO-SPB1, a tilted mode for EAS observing with a larger atmospheric footprint, and a sky mode to characterize the telescope using stars. Coupled with a SiPM array, the tilting would also enable measurements of background levels around the direction of the earth's limb as a precursor to searches for cosmogenic tau neutrinos.

Acknowledgment: This work was partially supported by NASA grants NNX13AH54G, NNX13AH52G, the French Space Agency (CNES), the Italian Space Agency through the ASI INFN agreement n. 2017-8-H.0, the Italian Ministry of Foreign Affairs and International Cooperation, the Basic Science Interdisciplinary Research Projects of RIKEN and JSPS KAKENHI Grant (22340063, 23340081, and 24244042), and the Deutsches Zentrum für Luft und Raumfahrt, and the 'Helmholtz Alliance for Astroparticle Physics HAP' funded by the Initiative and Networking Fund of the Helmholtz Association (Germany). We acknowledge the NASA Balloon Program Office and the Columbia Scientific Balloon Facility and staff for extensive support, the Telescope Array Collaboration for the use of their facilities in Utah. We thank the Wanaka airport staff and manager Ralph Fegan. We also acknowledge the invaluable contributions of the administrative and technical staffs at our home institutions.

\section{References}

[1] D.J. Bird et al., The Atrophysics Journal 424, 491 (1994).

[2] T. Abu-Zayyad et al., Nucl. Inst. Meth A450, 253 (2000).

[3] J. Abraham et al., [Pierre Auger Collaboration], Nucl. Inst. Meth A789, 172 (2015).

[4] H. Tokuno et al., [TA Collaboration], Nucl. Inst. Meth A676, 54 (2012).

[5] R. Benson, J. Linsley, Satellite observation of cosmic ray air showers in Proc 17th ICRC p. 8, (Paris), (1981).

[6] F. W. Stecker et al., Nucl. Phys B 136C, 433-438 (2004).

[7] J.F. Krizmanic et al., Optimization of the Orbiting Wide-angle Light Collectors (OWL) Mission for Charged-Particle and Neutrino Astronomy in Proc 33rd ICRC, (Rio De Janeiro), (2013) arXiv:1307.3907.

[8] A. Haungs, G. Medina-Tanco and A. Santangelo, Experimental Astronomy 40, 1 (2015).

[9] M. Casolino for the JEM-EUSO Collaboration, The EUSO missions to study UHECR from space: status and perspectives in Proc 35th ICRC, (Busan), PoS(ICRC2017)370 (2017).

[10] A. Olinto et al., Overview of POEMMA Science Goals in Proc 35th ICRC, (Busan), PoS(ICRC2017)542 (2017) arXiv:1708.07599.

[11] https://earth.nullschool.net.

[12] P. von Ballmoos et al., [JEM-EUSO Collaboration], General overview of EUSO-balloon mission in Proc. 34th ICRC, (The Hague), PoS(ICRC2015)322 (2015).

[13] M. Bertaina et al., [JEM-EUSO Collaboration], Preliminary results from the EUSO-Balloon flight in Proc. 34th ICRC, (The Hague), PoS(ICRC2015)358 (2015).

[14] S. Mackovjak et al., [JEM-EUSO Collaboration], Night Time Measurement of the UV Background by EUSO-Balloon in Proc. 34th ICRC, (The Hague), PoS(ICRC2015)685 (2015).

[15] J. Adams et al., [JEM-EUSO Collaboration], The calibration of EUSO-balloon using airborne light sources mounted to a helicopter in Proc. 34th ICRC, (The Hague), PoS(ICRC2015)580 (2015). 
[16] J. Eser et al., [JEM-EUSO Collaboration], EUSO-Balloon: Observation and Measurement of Tracks from a laser in a Helicopter in Proc. 34th ICRC, (The Hague), PoS(ICRC2015)638 (2015).

[17] Scientific American, Nov p32 (2014) and http://www.scientificamerican.com/article/cosmic-ray-telescope-flies-high/

[18] S. Bacholle et al., [JEM-EUSO Collaboration], The EUSO-SPB1 instrument in Proc 35th ICRC, (Busan), PoS(ICRC2017)384 (2017).

[19] Z. Plebaniak et al., J. HVPS system for *-EUSO detectors in Proc 35th ICRC, (Busan), PoS(ICRC2017)378 (2017).

[20] J. Bayer et al., [JEM-EUSO Collaboration], The trigger logic of EUSO-SPB1 and its expected performance in Proc 35th ICRC, (Busan), PoS(ICRC2017)443 (2017).

[21] G. Abdellaoui et al., [JEM-EUSO Collaboration], Nucl. Instr. \& Meth. A 866150 (2017) .

[22] G. Osteria et al., [JEM-EUSO Collaboration], The data processor system of EUSO-SPB1 in Proc 35th ICRC, (Busan), PoS(ICRC2017)461 (2017).

[23] W. Painter et al., Development of Silicon Photomultiplier Prototype for UHECR Fluorescence Detection from Space in Proc 35th ICRC, (Busan), PoS(ICRC2017)442 (2017).

[24] J.H. Adams et al., [JEM-EUSO Collaboration], Experimental Astronomy 40 p135 (2015).

[25] L. Allen et al., UCIRC: Infrared Cloud Monitor for EUSO-SPB1 in Proc 35th ICRC, (Busan), PoS(ICRC2017)436 (2017).

[26] I. Tabone et al., The WRF model contribution to the Cloud Top Height retrieval in EUSO-Balloon experiment in Proc 34th ICRC, (The Hague), PoS(ICRC2015)642 (2015).

[27] CSBF, Structural Requirements and Recommendations for Balloon Gondola Design, (OM-220-10-H) from https://www.csbf.nasa.gov

[28] P. Hunt et al., The JEM-EUSO global light system laser station prototype in Proc. 34th ICRC, (The Hague), PoS(ICRC2015)626 (2015).

[29] P. Hunt, MS Thesis, The JEM-EUSO Global Light System Laser Station Prototype, Colorado School of Mines, (2015).

[30] A. Cummings, MS Thesis, Field Testing of EUSO-SPB1: Logistics and first Results, Colorado School of Mines, (2016).

[31] L. Piotrowski et al., (with the JEM-EUSO collaboration), The EUSO-TA detector:status and performance in Proc 35th ICRC, (Busan), PoS(ICRC2017)374 (2017).

[32] J. Eser et al., [JEM-EUSO Collaboration], Preflight calibration and testing of EUSO-SPB1 in the lab and the desert in Proc 35th ICRC, (Busan), PoS(ICRC2017)457 (2017).

[33] M. Malek et al., Calibrating and Testing EUSO-SPB in Flight using a Laser and LEDs on an Aircraft in Proc 35th ICRC, (Busan), PoS(ICRC2017)448 (2017).

[34] F. Fenu et al., [JEM-EUSO Collaboration], Expected number of Extensive Air Showers observable by EUSO-SPB1 in Proc 35th ICRC, (Busan), PoS(ICRC2017)426 (2017).

[35] L. Wiencke et al., [JEM-EUSO Collaboration], EUSO-Balloon mission to record extensive air showers from near space in Proc. 34th ICRC, (The Hague), PoS(ICRC2015)631 (2015).

[36] J. Adams et al., White paper on EUSO-SPB2 arXiv:1703.04513v2. 